\title{
Identification of nanoscale localized strain in 2D transition metal dichalcogenide hybrid architectures through scanning transmission electron microscopy
}

Todd Brintlinger $^{1}$, Tomojit Chowdhury ${ }^{2}$ and Thomas Kempa ${ }^{2}$

${ }^{1}$ U.S. Naval Research Laboratory, United States, ${ }^{2}$ Johns Hopkins University, United States

Tuning the dimensionality of nanoscale materials is one route towards accessing exceptional and highly tunable properties [1-3]. An equally important approach involves the assembly of advanced hybrid architectures from discrete nanoscale components [4], often with different dimensionalities, compositions, and phases. The interfaces and interactions between these components gate the properties of the overall hybrid architecture and must be thoroughly understood to predict and design systems with new and emergent properties.

In this work, we drape 2D MoSe2 monolayers over single-crystal silicon nanowires (NW). Both materials are grown using a chemical vapor deposition process with MoO3, Se, and $\mathrm{SiH} 4$ precursors. The MoSe2 monolayers are sufficiently mechanically robust upon transfer over Si NWs spanning micron-sized holes in standard TEM grids (Fig. 1). Under high angle annular dark field scanning transmission electron microscopy (HAADF-STEM) characterization, we observe extensive and localized regions of strain in the region where the MoSe2 monolayers interface a facet of the $100 \mathrm{~nm}$ diameter Si NW. Near-field optical characterization reveals a photoluminescence (PL) peak characteristic of the expected MoSe2 "A" at 1.57 $\mathrm{eV}$. Notably, our efforts reveal an anomalous PL peak at $1.38 \mathrm{eV}$ that is localized to the aforementioned strained regions near the NW-monolayer interface [5]. To better understand and describe this peak's origins, we use aberration-corrected HAADF-STEM to determine the atomic structure of the MoSe2 near the Si NW.

Figure 2 shows portions of a single-crystal Si NW and a 1H-MoSe2 monolayer. HAADF-STEM reveals the presence of a highly localized region of strain near the NW-monolayer interface, and Fast Fourier Transforms (FFT) of specific regions-of-interest (ROIs) show that strains can approach almost 5\%. The strain increases monotonically within a 3-7 nm region near the $\mathrm{NW}$, and masking and inverse FFT operations show clear differences in the both the overall strain and its extent as the probe moves from the unstrained monolayer to the much thicker NW. While we can, of course, not completely discount the parallax of a 'draped' monolayer sheet in creating the appearance of strain, the concomitant appearance of strain-related PL shifts provides evidence for the presence of appreciable lattice strain. Thus, we identify the origin of the $1.38 \mathrm{eV}$ exciton as originating from this nanostrained interfacial region. 


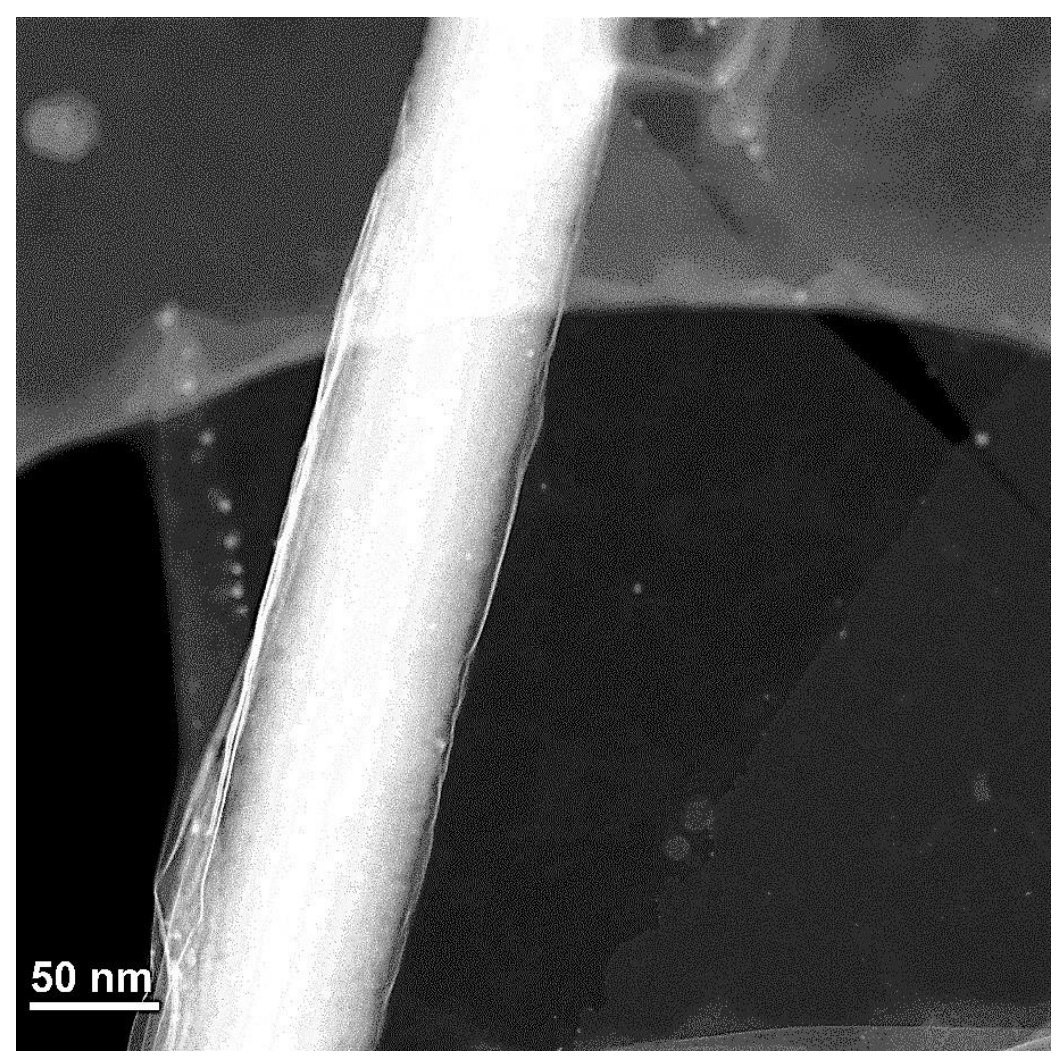

Figure 1. Figure 1: High-angle annular dark-field scanning transmission electron microscope (HAADFSTEM) image of a Si nanowire and MoSe 2 monolayer assembly spanning a $\sim \mu$ m circular hole. The monolayer is draped over the nanowire. Vacuum is on the left, and the right edge of the NW coming into contact with the monolayer is used for the analyses shown in Fig. 2.

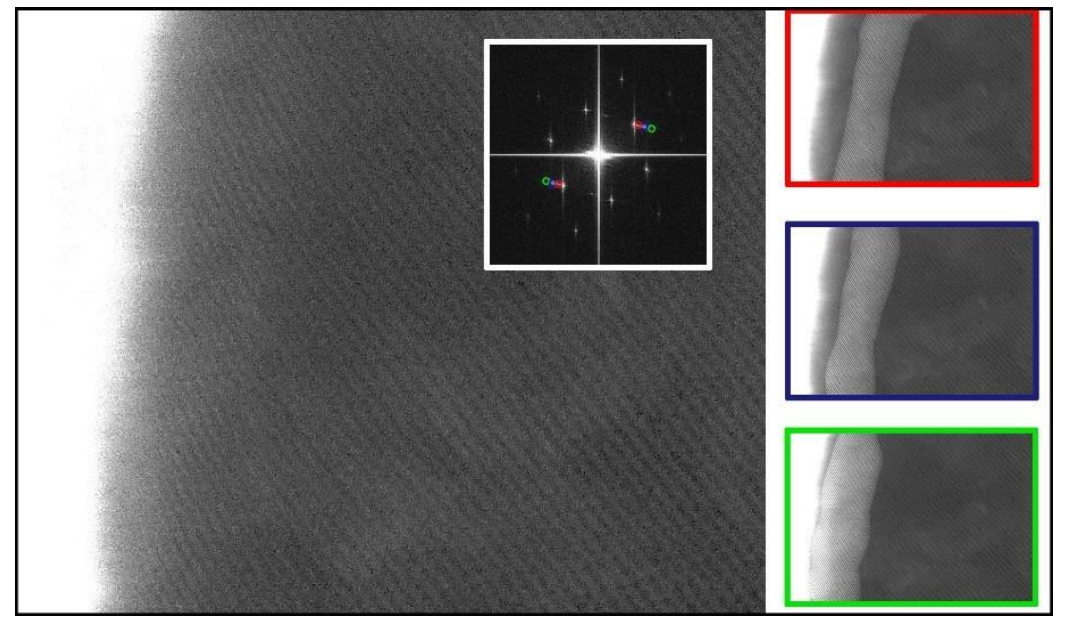

Figure 2. Figure 2: HAADF-STEM of the Si NW edge region within Fig. 1 with FFTs shown as insets. Red, blue, and green spots (in FFT) are used to define masks for the inverse FFTs with thresholds applied to produce the red, blue, and green boxed images. These data show the increasing level of strain in the monolayer as it nears the NW. 


\section{References}

[1] Lee, C.-H. ; Lee, G.-H. ; van der Zande, A. M. ; Chen, W.; Li, Y. ; Han, M. ; Cui, X.; Arefe, G. ; Nuckolls, C. ; Heinz, T. F. ; Guo, J.; Hone, J. ; Kim, Nat. Nanotechnol. 2014, 9, p. 676.

[2] Unuchek, D. ; Ciarrocchi, A.; Avsar, A. ; Watanabe, K. ; Taniguchi, T. ; Kis, A. Nature 2018, 560, p. 340.

[3] Castellanos-Gomez, A. ; Roldán, R. ; Cappelluti, E. ; Buscema, M. ; Guinea, F. ; van der Zant, H. S. J. ; Steele, G. A. Nano Lett. 2013, 13, p. 5361.

[4] Chowdhury, T. ; Sadler, E. C. ; Kempa, T. J. Chem. Rev. 2020, 120, 12563

[5] Jariwala, D.; Marks, T. J.; Hersam, M. C. Nat. Mater. 2017, 16, p. 170. 